\title{
Mechanism Analysis on Rotating Fiber for Particles Removal
}

\author{
Ling Shi*, Yufeng Chang and Hui Zhang \\ Hubei Key Laboratory of Industrial Fume \& Dust Pollution Control (Jianghan University), RPC Wuhan 430056 \\ ${ }^{*}$ Corresponding author
}

\begin{abstract}
Fiber filtration is an important technology to control fine particulates emission and to protect human health. The rotating interception efficiency conception is given according to a geometric model. The single and composite mechanisms about isolate rotating fiber filtration are analyzed, that mainly include inertia impaction, interception and diffusion. The experimental results, which rotating fiber pan is made of array rotating fiber, also are shown that the fractal efficiency curve is $v$-shape at constant rotating speed and constant gas velocity. Both theory stimulation efficiency and experimental results are reasonable coincident.
\end{abstract}

Keywords-rotating fiber; fiber filter; removal of fine particulates; aerosol filtration

\section{INTRODUCTION}

Fiber filtration is more simple and economical method to remove aerosol particulates from gas stream for industry application. It has one hundred histories on fiber filtration theory and technology, since S.K.Priedlander [1] put forward the filtration theory efficiency on isolated fiber. following some researchers also achieved[2-4]. Shi and Shusuke Nakajima[5,6] put forward different way of rotating fiber filter, respectively. Due to the complexity of filtration theory, fiber filtration theory lags behind fiber filtration technology development. So far, isolated fiber filtration theory is only relatively developed. Inertia, interception and diffusion are considered as main mechanism of removal of aerosol particles for isolated fiber.

The isolated fiber mechanism of particles removal may be demonstrated in Figure.1. As the gas flow perpendicularly through the static fiber, the aerosol particulates are removed. As all filters are composed of individual fiber, an understanding of the mechanisms by which the particles are removed on isolated cylinders and the flow pattern around them is of fundamental importance. It is clearly that the flow field and particulates motion around fiber may be acquired by solving N-S equation directly, but it is relatively complicated. So before 1959, the most challenging research is to acquire satisfactory flow velocity formula about the flow field around the isolated cylinder. Lamb and Davies $[7,8]$ have obtained the velocity field around the stationary cylinder which is perpendicular to flow, but the assumption is too simplifying,. After 1959, the research is focus on acquiring packed density formula which affects the removal efficiency. In this period, Kuwabara's [9] theory about the velocity field around the cylinder is widely accepted. So, many removal efficiency of fiber filtration are developed based on the Kuwabara's flow function.

The present paper gives a new kind of fiber filtration named as rotating fiber which removes the particles by rotating fiber cutting the particles-laden gas at vertical to the airflow. The difference between rotating fiber and conventional fiber filter is that the cutting gas flow mechanism is add to others mechanism. So the cleaning isn't necessary due to particle cohered on the fiber could be separated under rotating centrifugal force, at the same time, the rotating fiber filtration can achieves high efficiency and low pressure loss. Its prospective application might also be mist eliminator for water droplets and oil droplets adding to the particle removal.
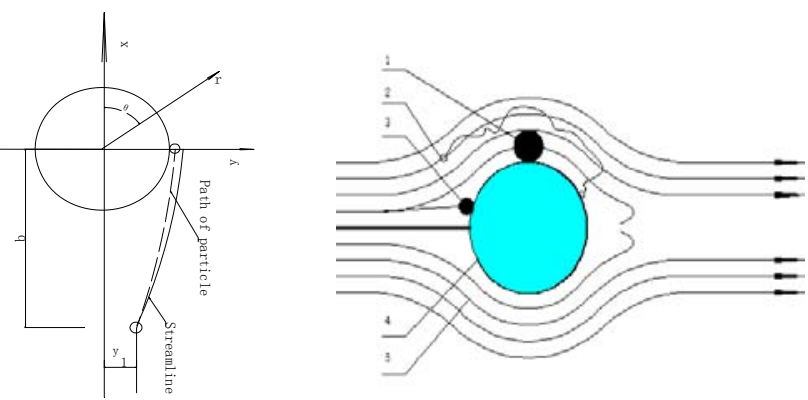

FIGURE I. ISOLATED FIBER FILTRATION MODEL

1.interception 2.diffusion 3. inertial impaction 4. section of fiber 5. stream line

At this work, the rotating fiber geometric model is shown, its aim is that the different between classic fiber filtration and rotating fiber are explained, firstly. Then the mechanism of rotating fiber filtration is analyzed, its aim is to determine the filtration efficiency under inertia, interception and diffusion action. Last, theory analysis and experimental results are shown.

\section{ROTATIN FIBER GEOMETRIC MODEL AND ANALYSIS}

A new type of filtration as "rotating fiber" was put forward, which removes aerosol particles by the fiber's rotating motion perpendicular to the direction of gas flow. Figure 2 shows the geometric model of rotating fiber. It is obvious that the rotating fiber cut the flow perpendicularly, so the probability of the particles captured is becoming big. Compare to the static fiber, rotating fiber has advantages on accelerating particles-to-fibers motion by centrifugal force. Following consequence is higher in collection efficiency and lower in pressure drop. 


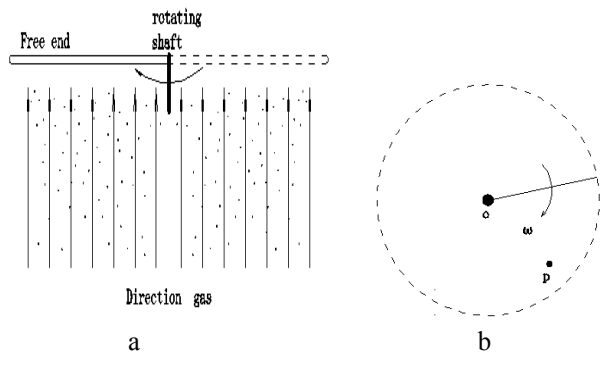

FIGURE II. ROTATING MODEL

a. front view b. top view

As referenced, the stationary isolate fiber efficiency is presented as formula (1) [9]

$$
\eta_{i}=\frac{y_{1}}{r_{s}}
$$

Where $r_{s}$ is fiber radius, $y_{1}$ is half width of interception in Figure.1 (a).

To obtain the value of $y_{1}$, the velocity around the fiber is necessary. The flow field can be given by Kuwabara's flow function in formula (2)[9].

$$
\psi(r, \theta)=\frac{v_{0} r}{2 K u}\left[2 \ln \frac{r}{r_{0}}-1+\alpha+\frac{r^{2} s}{r^{2}}\left(1+\frac{\alpha}{2}\right)-\frac{c r^{2}}{2 r_{s}^{2}}\right] \sin \theta
$$

Where $\mathrm{Ku}$ is Kuwabara hydrodynamic constant, and $K u=-\frac{1}{2} \ln \alpha+\alpha-\frac{1}{4} \alpha^{2}-\frac{3}{4}, \mathrm{v}_{0}$ is upstream velocity, $\mathrm{r}$ is the distance from the surface of fiber, $r_{s}$ is the radius of fiber, $\alpha$ is packed density. Adding to some constant, SI unit is adopted in all formulas, so they is not listed out one by one.

Above single fiber's important conclusion will also be applied in rotating fiber.

As shown in Figure.2, we will assume that effects of fiber length and neighbor fibers are omitted and airflow is perpendicular to the fiber.

\section{A. Transverse Interception Efficiency of Rotating Fiber}

The interception efficiency of single rotating fiber is that the rotating fiber cuts the airflow in transverse direction at a steady rotating angle $\omega$. The efficiency is defined as formula( 3$)$.

$$
\eta_{i}=\frac{V_{s}}{V}
$$

Where $V_{s}$ is the swept volume of isolated fiber with diameter $d_{f}$ at unit time, $V$ is the gas volume which flows the section with radius by length fiber at flow velocity $\mathrm{v}_{0}$.

Vs and V are expressed as following formula (4)and (5).

$$
\begin{gathered}
V_{S}=\pi D_{s} n\left(2 d_{p}+L\right)^{2} \\
V=\pi v_{0} L^{2}
\end{gathered}
$$

Where $d_{p}$ is the diameter of particulates, $D_{s}$ is the diameter of fiber, $\mathrm{L}$ is length of fiber, $\mathrm{n}$ is rotary number.

The efficiency of single rotating fiber versus the particle diameter, which is rotating fiber cutting particles-laden airflow, can be expressed as formula (6 ) under previous statements assumption condition.

$$
\eta_{s}=\frac{D_{s}}{v_{0}} \frac{\omega}{2 \pi}\left(\frac{2 d_{p}}{L}+1\right)^{2}
$$

\section{B. Longitudinal Direction Interception Efficiency of Rotating Fiber}

Stechina and Fuchs[3] gave the expression for isolated fiber interception efficiency by defined the interception parameter R, which is given in formula (7). Here the longitudinal direction interception efficiency is adopted, and it is expressed in formula (8) [10].

$$
R=\frac{d_{p}}{2 * r_{s}}
$$

$$
\eta_{l}=\frac{(1-\alpha) R^{2}}{K u(1+R)}
$$

Where $\mathrm{R}$ is the interception parameter, $\mathrm{Ku}$ is hydrodynamic constant, $\alpha$ is packed density.

\section{Gravitational Setting Efficiency}

Some particulates may settle on the surface of fiber under the force of gravity. For a horizontal cylinder transverse to the flow, the gravitational settling efficiency can be expressed by formula (9) [11].

$$
\eta_{S t}{ }_{k}=\frac{1}{18} \frac{C_{c} D_{p}{ }^{2}}{\mu}\left(1-\frac{\rho}{\rho_{p}}\right) g \frac{1}{v_{0}}=\frac{v_{t}}{v_{0}}
$$

Where $\mathrm{v}_{\mathrm{t}}$ is the gravity terminal setting velocity, $\rho$ is the density of air, $\rho_{p}$ is the density of particulates, $g$ is the acceleration of gravity.

It is worth noting that the gravity affection usually is omitted, unless the diameter of particulate is big. So the gravity setting also is omitted in this work. 


\section{Diffusion Efficiency}

Here, the expression is still adopted under Kuwabara's flow field, the diffusion efficiency is given by the following formula (10) [12]

$$
\eta_{D}=0.6396\left(\frac{1-c}{K u D v_{0}}\right)^{1 / 3}\left(\frac{k T C}{\mu d p}\right)^{2 / 3}
$$

Where $\mathrm{D}$ is diffusion parameter, $\mathrm{T}$ is thermodynamic temperature, $\mathrm{C}$ is Cunningham correction factor, $\mu$ is aerodynamic viscosity coefficient.

\section{E. Inertial Efficiency}

When a particulate is consider as a point mass, the particulate in front of cylinder can be either captured or escape from the flow due to inertial impaction. Inertial impaction is decided by Stokes number which is the ratio the inertial force to viscous force efficiency. The inertial efficiency may be expressed by formula (11) according to the Stechkina's study[3].

$$
\eta_{S t_{k}}=\frac{S t_{k}{ }^{2}}{\left(S t_{k}+0.25\right)^{2}}
$$

Where $\mathrm{St}_{\mathrm{k}}$ is stokes number, $S_{t_{k}}=\frac{\rho_{p} d_{p}^{2} v_{0}}{9 \mu d_{c}}, \mathrm{~d}_{\mathrm{c}}$ is the diameter of the interception body.

\section{F. Overall Efficiency}

The overall efficiency of rotating fiber can be expressed by formula (12) according to the probability

$$
\eta_{T}=1-\sum\left(1-\eta_{i}\right)
$$

Where $\eta_{i}$ is isolated mechanism fractal efficiency.

Figures 3 shows the single rotating fiber stimulation overall fractal efficiency curve by formula 12. As shown in Figure.3, at range of smaller particles diameter the efficiency is decrease with particles diameter increase, and at range of larger particles diameter the efficiency is increase with particles diameter, the most small efficiency is during of $0.1 \mu \mathrm{m}$ to $1 \mu \mathrm{m}$. It is possible that the diffusion mechanism plays a part mainly within smaller particles diameter range, and the inertial and interception mechanism plays a part mainly within larger particles diameter range.

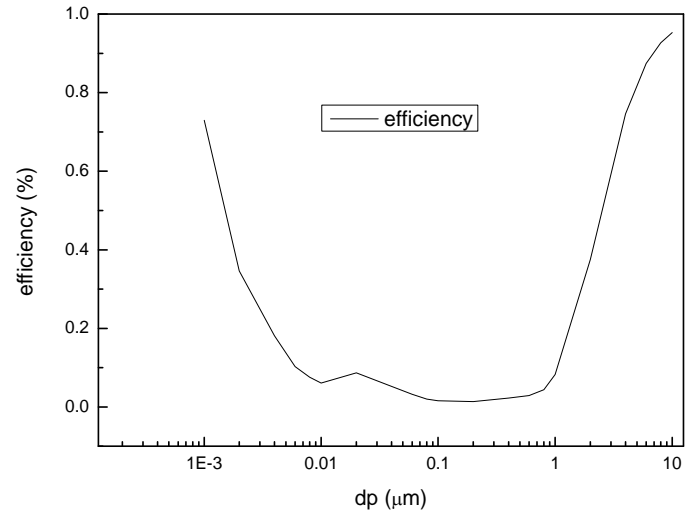

FIGURE III. OVERALL EFFICIENCY STIMULATION CURVE

\section{EXPERIMENTS}

To contrast with the theory analysis of rotating fiber a experimental setup is built. The schematic diagram of experimental setup is presented in Figure.4. It is consist of the duct, dust generation, main body (included rotating fiber pan), and fan. The frame of the rotating fiber pan is made of the stainless steel and the diameter of cotton fibers is $0.02 \mathrm{~mm}$. The rotating fiber pan is driven by adjusted speed DC motor. The median diameter of particulates is $1.4 \mu \mathrm{m}, 2.4 \mu \mathrm{m}, 5.4 \mu \mathrm{m}, 7.8 \mu \mathrm{m}$ and $9.7 \mu \mathrm{m}$, respectively. The airflow velocity is $2 \mathrm{~m} / \mathrm{s}$. The weight is utilized to obtain the collection fractal efficiency of rotating fiber.

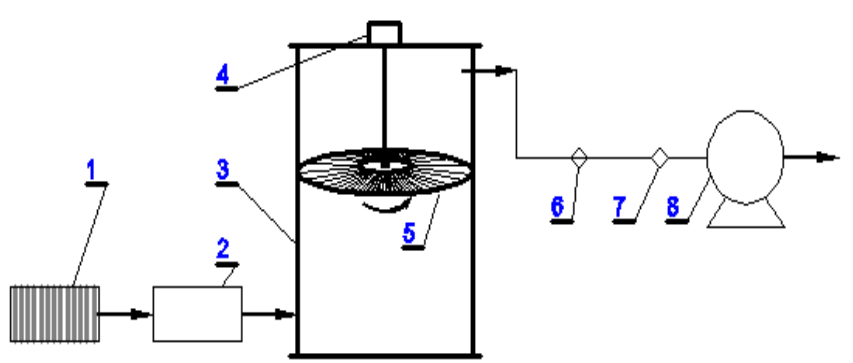

FIGURE IV. SCHEMETIC EXPERIMENTAL SYSTEME

1 air filter 2 dust generation 3 main body 4 rotating motor 5 rotating fiber pan 6 concentration measuring hole 7 pressure measuring hole 8 fan

The result is presented in Figure.5. The minimum collection efficiency is at median diameter of $5 \mu \mathrm{m}$ under the rotation speed of 1000rpm. When the diameter of particulates is less than $5 \mu \mathrm{m}$, the collection efficiency deduced with the increasing of diameter, on the contrary when the diameter of particulates is less than $5 \mu \mathrm{m}$. The overall fractal efficiency is more than $94 \%$. It is obvious that the fractal efficiency curve is v-shape. The experimental change trend is consistent with the above theory stimulated curve. Since the rotating fiber special structure which is single layer array the exponential efficiency curve shape is not shown. Comparison the theory stimulation with the experimental curve, it can be found that the theory efficiency is low the experimental results, the cause is that the 
theory curve is single rotating fiber, however experimental results is achieved under multi-fiber condition.

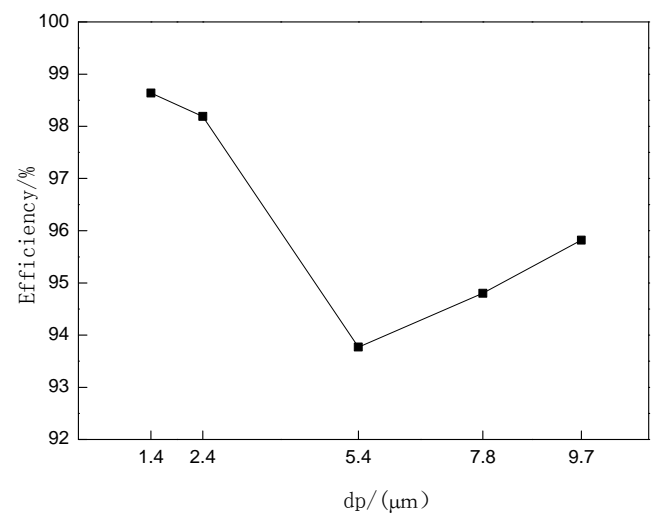

FIGURE V. EXPERIMENTAL EFFICIENCY CURVE

\section{CONCLUSIONS}

A new fiber filter technology was provided, in which the rotating fiber enhances the collection efficiency significantly by rotating cutting airflow, at the same time, the rotating fiber has self-cleaning ability due to fiber rotating .

Several mechanisms of rotating fiber for particulates removal are analyzed, and the efficiency of rotating fiber pan, which is made of rotating fiber array, also is demonstrated experimentally.

Comparing to efficiency of rotating fiber experimental results and theoretic stimulation, and change trend of two is the same basically.

\section{ACKNOWLEDGMENT}

The authors would like to thank Hubei province natural science foundation directive project(2016CFA093) and Hubei Key Laboratory of Industrial Fume \& Dust Pollution Control open project (HBIK2017-05).

\section{REFERENCES}

[1] S.K.PRIEDLANDER, 'Theory of Aerosol Filtration," Industrial and Engineering Chemistry,vol.50,No.8, ,pp:1161-1164,1959.

[2] Gordon M. Bragg and Bruce M. Pearson, "A Model of Aerosol Filtration by Fibrous Filters," Ind.Eng.Chem. Process Des, Vol.18,No.1 pp:171178,1979

[3] Stechkina, I. B., Kirsh, A. A, Fuchs. N. A, "Studies on Fibrous Aerosol Filters-IV Calculation of Aerosol Depostion in Model Filters in the Range of Maximum Penetration, Ann. Occup. Hyg. Vol.12:1-8, 1969.

[4] Stechkina, I.B. and Fuchs,N.A." Studies on Fibrous Aerosol Filters-I Calculation of Diffusion Deposition of Aerosol in Fibrous Filters," Aerosol Sci. Technol. Vol.9,PP:59-64,1966.

[5] Shi Ling, Xingming Yu, "a kind of swirl flow and cutting precipitator, "patent, ZL20141023212.9.

[6] Shusuke Nakajima, Mikio Kumita, Hiroaki Matsuhashi, Hidenori Higashi Takafumi Seto \& Yoshio Otani, "Centrifugal Filter for Aerosol Collection," Aerosol Science and Technology Vol.49,PP:959-965,2015.

[7] Lamb, H., Hydrodynamics, 6th ed., Cambridge University Press, Lodon, 1932, pp 614.
[8] Davies, C.N., Air filtration, 1st ed, London and New York Academic Press 1973, pp53-54,

[9] Kuwabara, s., "The Force experienced by Randomly Distributed Parallel Circular Cylinders or Sphere in a viscous Flow at small Reynolds N umber, " J. Phys. SocJpn,14:527, 1959.

[10] K. W. Lee and B. Y. Liu, "Theoretical Study of Aerosol Filtration by Fibrous Filters, " Aerosol Science and Technology Vol.1,No.2, pp:1471161,1982 .

[11] Yoshioka,N., Kanaoka, C. Emi, H., and Yasunami, M, "Collection Efficiency of Aerosol by an Isolated Cylinder-Gravity and Inertial Predominant Region," Kagaku Kougaku, Vol.36:313-319,1972.

[12] Martin C.. Air pollution control theory, 1st ed, New York: Mcgrw Hill 1976, PP362-373 